\title{
On-Orbit Self-Compensation of Satellite Optics Using Spatial Light Modulator
}

\author{
By Norihide MIYAMURA ${ }^{1)}$ \\ ${ }^{1)}$ Research Center for Advanced Science and Technology, The University of Tokyo, Tokyo, Japan
}

(Received July 17th, 2009)

\begin{abstract}
We propose an adaptive optics system for a lightweight remote sensing sensor. The phase diversity (PD) technique, in which known wavefronts (Phase Diversity) are applied to the optics and the inherent aberrations are estimated using the acquired images without a priori information, is a key to realizing the system. For the reduction of computing cost and the enhancement of the estimation accuracy of aberration, a spatial light modulator (SLM) is adopted not only for wavefront compensator but also for PD generator. The SLM produces arbitrary "aberration modes" that are each represented by a Zernike polynomial. Therefore, optimal phase diversities are applied to the optical system and particular modes are effectively obtained, which makes it possible to overcome the conventional PD generated by defocusing that describes only quadratic form and lacks information of a particular mode. In order to solve the complex inverse problem of phase diversity with low computing cost, a general regression neural network (GRNN) is used. Moreover, principal component analysis compresses the input data for GRNN by extracting information from collected images in Fourier space, and reduces computation cost considerably. The performance is validated by numerical simulation, and the result of experiment using SLM is described.
\end{abstract}

Key Words: Adaptive Optics, Spatial Light Modulator, Phase Diversity, Neural Network, Principal Component Analysis

\section{Nomenclature}

$a_{j} \quad$ :aberration parameter

$A \quad$ :binary pupil function

$b_{j} \quad$ :phase diversity parameter

$i \quad$ :image

$M \quad$ :merit function

$n \quad$ :noise

$o \quad$ :object

p : feature vector

$P \quad$ :generalized pupil function

$s \quad$ :point spread function

$(u, v) \quad$ :spatial frequency coordinates

$(x, y)$ :focal plane coordinates

$Z_{\mathrm{j}} \quad$ :Zernike polynomials

$\theta \quad$ :phase diversity

$\Phi$ :wavefront aberration function

$(\xi, \eta) \quad$ :pupil plane coordinates

Subscripts

$0 \quad$ :in-focus value

$\mathrm{k} \quad: k$ th phase diversity value

i :image plane

o :object plane

p :pupil plane

\section{Introduction}

Since satellite remote sensing has the advantages of wide area and periodic observation compared with airplane and ground observation, high resolution in both spatial and spectral regions is required. Accordingly, an optical system with large aperture and precise alignment must be prepared.
Furthermore, satellite sensors are subjected to severe environments such as launch vibration and thermal stress in an extremely wide temperature range in orbit. These situations result in optical performance degradation due to the misalignment of optical elements. Traditional sensors use a rigid supporting structure to avoid the degradation of optical performance. However, it makes the sensors heavy and leads to high launch cost. Moreover thermal deformation could not be completely eliminated by the rigid structure. To realize high performance and a lightweight structure at the same time, on-orbit self-compensation of remote sensing optical sensors is necessary.

For ground telescopes, the adaptive optics system (AOS) has been used for wavefront compensation in astronomy. The AOS measures atmospheric turbulence and compensates it using a wavefront sensor, e.g., a Shack-Hartmann wavefront sensor, which needs a priori information that the observed target is the point source. For satellite remote sensing, a priori information is not available, and calibration of a wavefront sensor is difficult in orbit.

The phase diversity (PD) method ${ }^{1)}$ is a technique for estimating wavefront aberration on the basis of observed images instead of using wavefront sensors. Consequently, calibration problems related to wavefront sensors are solved. In the phase diversity method, known phase aberration is applied to the optical system and images are acquired; then wavefront aberration is estimated using the acquired images. In this method, the way of applying known aberration to the system is one of the essential issues. In the traditional approach, the only way to generate phase diversity is to defocus the detector ${ }^{2}$.

Recently, a spatial light modulator (SLM) that can control wavefront by changing the optical path difference in 
two-dimensional pixel structures was developed ${ }^{3-6)}$. By means of SLM, it is possible to generate an arbitrary wavefront shape and an arbitrary number of phase diversity. Accordingly, the coverage of aberration to be estimated is extended and the accuracy of estimated wavefront aberration is improved.

However, PD method requires complicated image processing to estimate aberration from observed images compared with traditional wavefront sensors. For onboard estimation of wavefront aberration in a poor resource such as a small satellite, computation cost may be the restriction in realizing the self-compensating system. In this study, a neural network is used to solve the complicated inverse problem. Furthermore, principal component analysis (PCA) is used for the preprocessing of the neural network to compress the information.

In this paper, we describe a self-compensating optical system in which wavefront aberration is estimated using the observed images and compensated using the SLM. A numerical simulation and experiment results are described.

\section{Mathematical Model}

\subsection{Formulation of optical systems using phase diversity method}

The estimation problem of wavefront aberration is formulated as a well-posed inverse problem by the phase diversity method ${ }^{1)}$. Phase diversity is defined as a known wavefront aberration that is intentionally applied to the optical system as a priori information, and the images obtained with phase diversities are defined as diversity images.

The performance of optical systems is formulated on the basis of the scalar diffraction theorem. The $k$ th observed image $i_{\mathrm{k}}(x, y)$, which is obtained with $k$ th phase diversity $\theta_{\mathrm{k}}(x, y)$, is expressed as a convolution of the $k$ th point spread function (PSF) $s_{\mathrm{k}}(x, y)$ and an observed object $o(x, y)$ in the focal plane under isoplanatic and quasi-monochromatic conditions $^{7}$,

$$
i_{k}(x, y)=s_{k}(x, y) * o(x, y)+n_{k}(x, y) .
$$

$s_{\mathrm{k}}(x, y)$ is expressed by the Fourier transform of the $k$ th generalized pupil function $P_{\mathrm{k}}(\xi, \eta)$ in the case of noise $n_{\mathrm{k}}(x, y)$ being negligible,

$$
s_{k}(x, y)=\iint P_{k}(\xi, \eta) \exp (-i 2 \pi(\xi x+\eta y)) d \xi d \eta .
$$

The generalized pupil function is expressed using binary pupil function $A(\xi, \eta)$ that is unity within the aperture and zero otherwise, and wavefront function that consists of a wavefront aberration and the $k$ th phase diversity,

$$
P_{k}(\xi, \eta)=A(\xi, \eta) \exp \left(\Phi(\xi, \eta)+\theta_{k}(\xi, \eta)\right) .
$$

The wavefront aberration is defined as the wavefront deflection between an ideal spherical wavefront and the actual wavefront. $\Phi(\xi, \eta)$ and $\theta_{\mathrm{k}}(\xi, \eta)$ are expanded on a finite set of Zernike polynomials $Z_{\mathrm{j}}(\xi, \eta)^{8,9)}$ :

$$
\begin{aligned}
& \Phi(\xi, \eta)=\sum_{j=1}^{J} a_{j} Z_{j}(\xi, \eta), \\
& \theta_{k}(\xi, \eta)=\sum_{j=1}^{J} b_{j} Z_{j}(\xi, \eta),
\end{aligned}
$$

where each $Z_{\mathrm{j}}(\xi, \eta)$ is independent of others in the circular aperture. In this work, wavefront aberration represented by $Z_{\mathrm{j}}(\xi, \eta)$ is defined as the "aberration mode". Low-dimensional Zernike polynomials correspond to Seidel aberrations; the 4th, 5th, and 8th aberration modes correspond to astigmatism, defocus, and coma, respectively, in the standard Zernike polynomials order ${ }^{10)}$. An aberration vector $\boldsymbol{a}$ and $k$ th phase diversity vector $\boldsymbol{b}_{\mathrm{k}}$ are defined by,

$$
\begin{gathered}
\boldsymbol{a}=\left(a_{1}, a_{2}, \ldots, a_{J}\right)^{T}, \\
\boldsymbol{b}_{\mathrm{k}}=\left(b_{1}{ }^{(k)}, b_{2}{ }^{(k)}, \ldots, b_{J}{ }^{(k)}\right)^{T} .
\end{gathered}
$$

The goal of the wavefront estimation is finding $\boldsymbol{a}$ using $i_{\mathrm{k}}$ and $\boldsymbol{b}_{\mathrm{k}}$ under the condition of an unknown observed object.

\subsection{Definition of unique function of aberration parameters}

In the traditional phase diversity method, aberration vectors are estimated by optimizing a cost function based on the least square approach $^{1,11)}$, which requires iterative calculation until the cost function is minimized.

An alternate merit function $M_{\mathrm{k}}(u, v)$ is defined in Fourier space using diversity images ${ }^{12,13)}$,

$$
\begin{aligned}
M_{k}(u, v) & =\frac{I_{0}{ }^{*}(u, v) I_{0}(u, v)-I_{k}{ }^{*}(u, v) I_{k}(u, v)}{I_{0}{ }^{*}(u, v) I_{0}(u, v)+I_{k}{ }^{*}(u, v) I_{k}(u, v)} \\
& =\frac{S_{0}{ }^{*}(u, v) S_{0}(u, v)-S_{k}{ }^{*}(u, v) S_{k}(u, v)}{S_{0}{ }^{*}(u, v) S_{0}(u, v)+S_{k}{ }^{*}(u, v) S_{k}(u, v)}
\end{aligned}
$$

where $I$ and $S$ are the Fourier transforms of $i$ and $s$, respectively, and $*$ denotes a complex conjugate. The Fourier transform of object $O(u, v)$ is canceled out from the numerator and denominator, so $M_{\mathrm{k}}(u, v)$ depends not on observed objects but on aberration parameters only. $M_{\mathrm{k}}(u, v)$ is unique for an aberration parameter, therefore, aberration vectors are estimated by learning the relationship between $M_{\mathrm{k}}(u, v)$ and aberration vectors.

\section{Estimation of aberration parameters from observed images}

The data processing flow to estimate aberration parameters using machine learning is shown in Fig. 1. The procedure consists of the training phase and the operation phase.
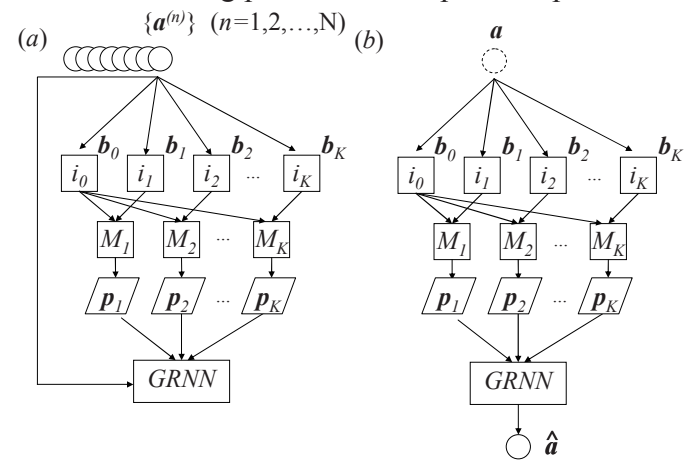

Fig. 1. Data processing flow in (a) training phase and (b) operation phase. 
In the training phase, aberration vectors $\left\{\boldsymbol{a}^{(\mathrm{n})}\right\}(n=1,2, \ldots, N)$ are prepared, and not only phase diversity $\left\{\boldsymbol{b}_{\mathrm{k}}\right\}$ but also $\left\{\boldsymbol{a}^{(\mathrm{n})}\right\}$ are artificially applied to the optical system using SLM for supervised training. Phase diversities should be selected to prevent degradation of the estimation accuracy of a particular aberration mode, which is described in section 3.1. For applied aberration vector $\boldsymbol{a}^{(\mathrm{n})}$ and phase diversity vector $\boldsymbol{b}_{\mathrm{k}}$, diversity image $i_{\mathrm{k}}(x, y)$ is acquired, then $M_{\mathrm{k}}(u, v)$ is calculated for each phase diversity vector.

The pixels on $M_{\mathrm{k}}(u, v)$ do not equally have information about wavefront aberration. Accordingly, it is not efficient to use all pixels to represent the relationship with aberration vectors. A pixel value on $M_{\mathrm{k}}(u, v)$ that includes information about aberration is defined as a feature vector $\boldsymbol{p}_{\mathrm{k}}$, which is used to represent the relationship.

The learning system is trained to represent the relationship between $\boldsymbol{a}^{(\mathrm{n})}$ and $\left\{\boldsymbol{p}_{\mathrm{k}}\right\}$ instead of the $\left\{M_{\mathrm{k}}(u, v)\right\}$ in order to reduce computation cost. PCA is used for extracting $\left\{\boldsymbol{p}_{\mathrm{k}}\right\}$, which is described in section 3.2. For the architecture of the learning system, the generalized regression neural network (GRNN), of which the response function of neurons consists of a radial basis function, is used for the estimation of aberration parameters ${ }^{12)}$. By means of GRNN, $\boldsymbol{a}$ is represented as a function of $\left\{\boldsymbol{p}_{\mathrm{k}}\right\}$.

In the operation phase, the unknown wavefront aberration $\boldsymbol{a}$ existing in optics is estimated. Here, the same phase diversity vectors as were applied in the training phase are used and $\boldsymbol{p}_{\mathrm{k}}$ is calculated by the same procedure as that in the training phase.

The estimated aberration parameter $\hat{\boldsymbol{a}}$ is calculated using

GRNN, the neuron weights of which were determined in the training phase.

\subsection{Phase diversity selection using spatial light modulator}

Each aberration parameter generates unique distribution patterns on $M_{\mathrm{k}}(u, v)$. This characteristic is used to estimate aberration parameters using observed images. When the change in $M_{\mathrm{k}}(u, v)$ owing to the change of the $i$ th aberration parameter is significantly smaller than that of the $j$ th aberration parameter, where $i \neq j$, the estimation accuracy of $a_{\mathrm{i}}$ is degraded compared with that of $a_{\mathrm{j}}$. Furthermore, when the noise level is not negligible compared with the change of $M_{\mathrm{k}}(u, v)$ owing to the change of $a_{\mathrm{i}}$, the estimation accuracy of $a_{\mathrm{i}}$ becomes worse.

Since $M_{\mathrm{k}}(u, v)$ is a function of not only $\boldsymbol{a}$ but also $\boldsymbol{b}_{\mathrm{k}}$, the estimation accuracy of $a_{\mathrm{j}}$ is improved by making the response of $M_{\mathrm{k}}(u, v)$ stronger against $a_{\mathrm{j}}$ by selecting phase diversity.

Figure 2 shows the strategy for phase diversity selection. First, phase diversity vector $\boldsymbol{b}_{1}$ is selected as an initial value and applied. Aberration parameter $a_{\mathrm{j}}$ is changed by $\Delta a$ in each time to evaluate the response of $M_{\mathrm{k}}(u, v)$ against $a_{\mathrm{j}}$,

$$
a^{(l)}=a^{(l-1)}+\Delta a,(l=1,2, \ldots, L) .
$$

The variance on each pixel is calculated for $\left\{M^{(1)}{ }_{\mathrm{k}}(u, v)\right\}$.
When the maximum variance is larger than a threshold $V$, the aberration parameter is considered to be estimated. In contrast, when the variance is smaller than the threshold, the aberration parameter is not estimated, so phase diversity is selected to change the response of $M_{\mathrm{k}}(u, v)$ against the aberration parameter. In this work, the phase diversity vector that has a non zero element of $b_{\mathrm{j}}$ is added to the phase diversity vectors $\left\{\boldsymbol{b}_{\mathrm{k}}\right\}$. For all aberration modes, the variance of $M_{\mathrm{k}}(u, v)$ is evaluated and then the phase diversity is added to the phase diversity vectors if it is necessary.



Fig. 2. Phase diversity selection flow.

\subsection{Principal component analysis for data extraction}

Since $M_{\mathrm{k}}(u, v)$ shows independent distribution against the aberration mode, the aberration vector is estimated by learning the relationship between them using GRNN. To represent the aberration information with a small amount of data, the independent component of $M_{\mathrm{k}}(u, v)$ is extracted using PCA, then the pixels on $M_{\mathrm{k}}(u, v)$, that contribute to each independent component are extracted as feature vectors and used to train the GRNN with the aberration vector. In this way, the input data size for GRNN is drastically reduced.

Before conducting PCA, $M_{\mathrm{k}}(u, v)$ for each phase diversity is collected using all training aberration vectors, as shown in Fig.1. The $i$ th $\mathrm{PC} z_{\mathrm{i}}$ is calculated as

$$
z_{i}^{(k)}=\sum_{j} w_{i j}^{\left({ }^{k}\right)} z^{\prime}{ }_{j}^{(k)},
$$

where $z_{\mathrm{j}}{ }^{(\mathrm{k})}$ denotes the $j$ th pixel value in $M_{\mathrm{k}}(u, v)$ and $w_{\mathrm{ij}}{ }^{(\mathrm{k})}$ denotes the weight of $x_{\mathrm{j}}^{(\mathrm{k})}$ for the $i$ th principal component. By means of PCA, the independent change on $M_{\mathrm{k}}(u, v)$ against the aberration mode is extracted as PC. In this case, the weight $w_{\mathrm{ij}}{ }^{(\mathrm{k})}$ represents the contribution of the pixels for the $i$ th PC. Accordingly, pixels that have large weights are used to 
represent the relationship between aberration vectors and observed images. GRNN is trained using the aberration vector and extracted data as training data.

\section{Numerical Simulation}

\subsection{Numerical model of optical system and wavefront aberration}

The specifications of numerical model are shown in Table 1. The telescope is implemented as a refractive optical system using a doublet, as shown in Fig.3. Optical aberration is induced by defocus and tilt of the lens. The maximum range of the aberration parameter for GRNN training is determined prior to the training phase, as shown in Table 2. These parameters are calculated in the case of $\pm 1.0 \mathrm{~mm}$ defocus and $\pm 1.0 \mathrm{deg}$ rotation of lens. The main aberration is distributed in $a_{4}, a_{5}$, and $a_{8}$, and other parameters have negligible values. This is because wavefront aberration based on defocus and rotation of the lens is limited to a low spatial frequency. The aberration vector and the phase diversity vector are represented as $\boldsymbol{a}=\left(a_{4}, a_{5}, a_{8}\right)^{\mathrm{T}} \quad$ and $\quad \boldsymbol{b}_{\mathrm{k}}=\left(b_{4}{ }^{(\mathrm{k})}, b_{5}{ }^{(\mathrm{k})}, b_{8}{ }^{(\mathrm{k})}\right)^{\mathrm{T}}$, respectively. The following sections describe the selection of phase diversity, the effect of PCA, and the end-to-end performance of the proposed self-compensating system.

Table 1. Preconditions of imaging system.

\begin{tabular}{|l|l|}
\hline F number & 4 \\
\hline Focal length & $400 \mathrm{~mm}$ \\
\hline Wavelength & $550 \mathrm{~nm}$ \\
\hline SNR & 30 \\
\hline
\end{tabular}



Fig. 3. Telescope model for numerical simulation.

Table 2.
\begin{tabular}{|l|c|l|}
\hline$a_{4}$ & \pm 0.259 & astigmatism \\
\hline$a_{5}$ & \pm 1.16 & defocus \\
\hline$a_{6}-a_{7}$ & - & \\
\hline$a_{8}$ & \pm 0.626 & coma \\
\hline$a_{9}-a_{10}$ & - & \\
\hline
\end{tabular}

\subsection{Phase diversity selection}

Phase diversities are selected by the method described in section 3.1. Initial phase diversity $\boldsymbol{b}_{1}$ is set to the defocus term represented by $\boldsymbol{b}_{1}=(0,0.5,0)^{\mathrm{T}}$. This is the same as that applied in the traditional phase diversity method.

Prior to training the GRNN, aberration parameters of $a_{4}, a_{5}$, and $a_{8}$, as supervised training data, are varied independently to evaluate the changes on $M_{1}(u, v)$. As shown in Fig. 2, the variances of the pixels on $M_{1}(u, v)$ are calculated when a single aberration parameter changes. The two-dimensional distributions of the variance for each aberration parameter are shown at the top of Fig. 4. The area where the pixel values are relatively large represents where $M_{1}(u, v)$ varies with the aberration parameter. According to the figure, the maximum amplitudes for the aberration parameters of $a_{4}, a_{5}$, and $a_{8}$ are $0.12,0.25$, and 0.09 , respectively. The variance of $M_{1}(u, v)$ with the change of $a_{8}$ is smaller than that with other aberration parameters. When the noise effect is not negligible, the change of $M_{1}(u, v)$ owing to $a_{8}$ is difficult to detect.

For the improvement of the response of the variance of $M_{1}(u, v)$ against the change of aberration parameter $a_{8}$, phase diversity, which is represented by $b_{8}$, is added to the phase diversity vectors as $\boldsymbol{b}_{2}=(0,0,0.5)^{\mathrm{T}}$ in accordance with the selection strategy described in section 3.1. The two-dimensional distribution of the variance of $M_{2}(u, v)$ for each aberration parameter change is shown at the bottom of Fig. 4. The maximum value of the amplitude for the aberration parameters of $a_{4}, a_{5}$, and $a_{8}$ are $0.09,0.15$, and 0.23 , respectively. The response of $M_{2}(u, v)$ against $a_{8}$ is considerably improved, but the responses against $a_{4}$ and $a_{5}$ are degraded compared with that with the phase diversity of $b_{5}$. Consequently, the required phase diversities are selected as $\boldsymbol{b}_{1}=(0,0.5,0)^{\mathrm{T}}$ and $\boldsymbol{b}_{2}=(0,0,0.5)^{\mathrm{T}}$. GRNN is trained using these two phase diversities.

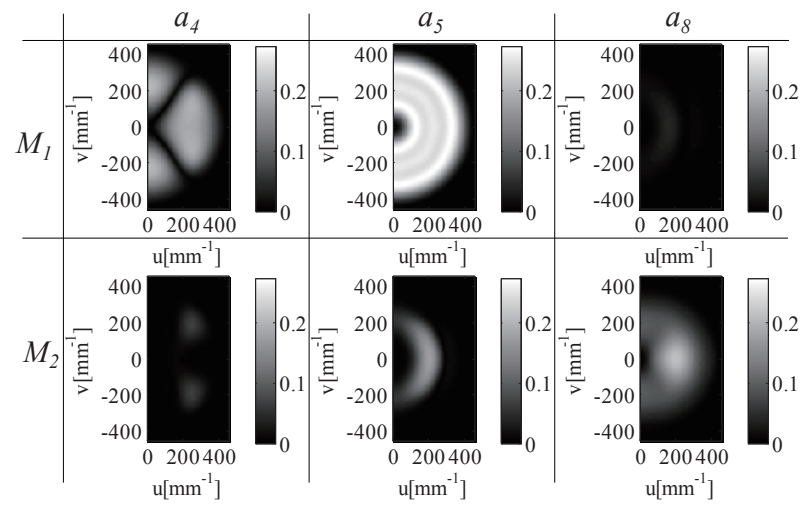

Fig. 4. Distributions of variances for $\left\{M^{(\mathrm{i})}{ }_{\mathrm{k}}(u, v)\right\} ; M_{1}$ for $\boldsymbol{b}_{1}=(0,0.5,0)^{\mathrm{T}}$ and $M_{2}$ for $\boldsymbol{b}_{2}=(0,0,0.5)^{\mathrm{T}}$.

\subsection{End-to-end self-compensation system performance}

Aberration parameters are estimated from observed images using the trained GRNN. The estimated aberration parameters for single PD, (a) $\boldsymbol{b}_{1}$, (b) $\boldsymbol{b}_{2}$, and (c) $\boldsymbol{b}_{1}$ and $\boldsymbol{b}_{2}$, are shown in Fig. 5. In case (a), standard deviations of estimated parameters for $a_{4}$ and $a_{8}$ are worse than that for $a_{5}$. In case (b), the standard deviation of the estimated parameter for $a_{8}$ improved. However, those for $a_{4}$ and $a_{5}$ became worse. In case (c), 
standard deviations of all estimated parameters improved significantly.

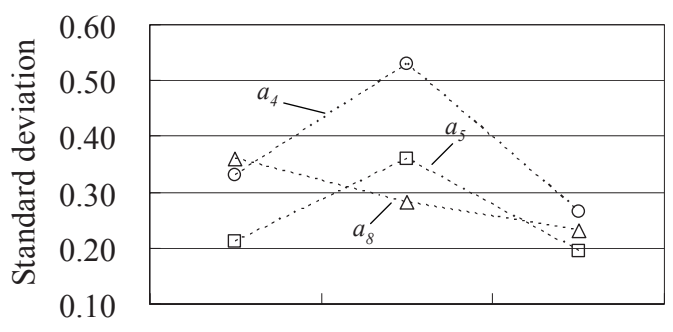

(a) (b)

(c)

Fig. 5. Variances of estimated aberration parameters for 100 trials: (a) $\boldsymbol{b}_{1}=(0,0.5,0)^{\mathrm{T}}$, (b) $\boldsymbol{b}_{2}=(0,0,0.5)^{\mathrm{T}}$, and (c) $\boldsymbol{b}_{1}$ and $\boldsymbol{b}_{2}$.

\section{Experiment}

As I described above, estimation accuracy of aberration parameters are improved by selecting optimal phase diversities. In this section, I describe the result of the wavefront compensation experiments. In the experiments, wavefront aberration was introduced by the defocus and tilt of conversing lens. The experiment configuration is shown in Fig. 6.

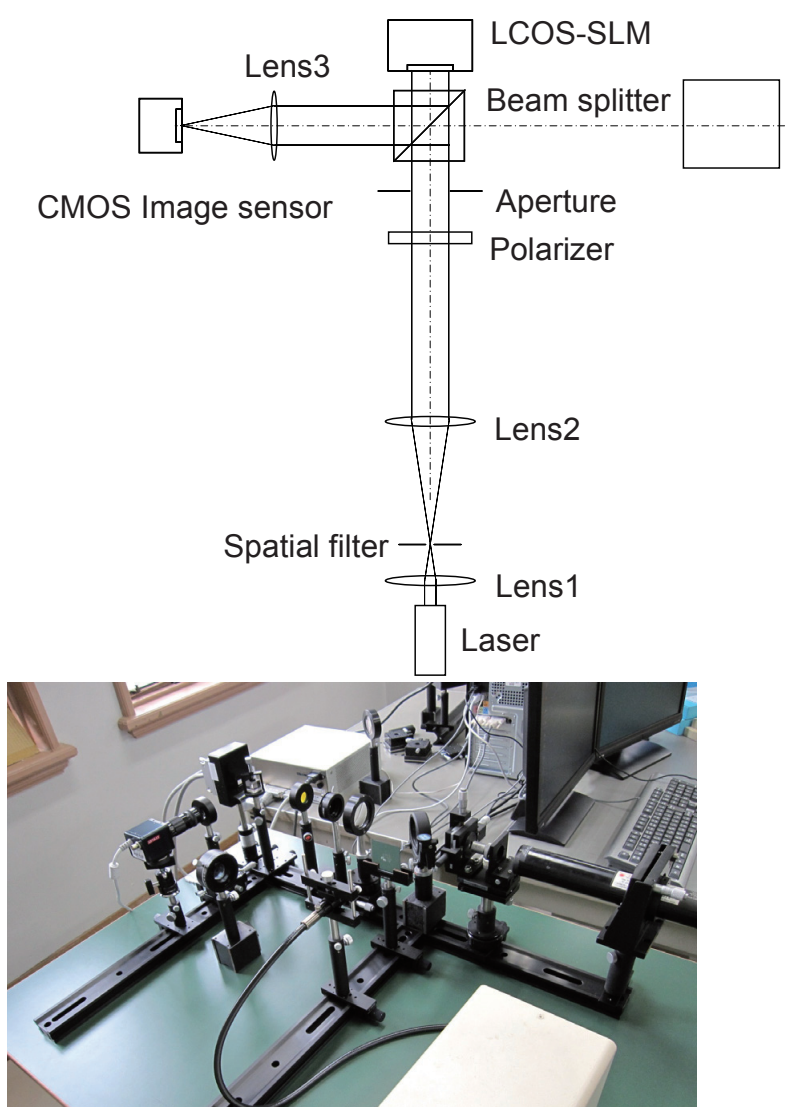

Fig. 6. Experiment configuration.

For simplification, a point source is generated using laser source with spatial filter. After collimating with lens 2, incident light is attenuated by polarizer, and aperture is used to adjust the incident flux to LCOS-SLM (Liquid Crystal on Silicon - Spatial Light Modulator) effective area. The modulated light is converged by lens 3 , and image is obtained at CMOS camera. In this configuration, lens 3 is defocused and rotated by $z-\theta$ stage to generate wavefront aberrations. The specification of LCOS-SLM and CMOS camera are shown in Table 3 and Table 4, respectively.

Table 3. The specification of the LCOS-SLM.
\begin{tabular}{|l|l|}
\hline pixels & $800 \times 600$ \\
\hline frame rate & $60 \mathrm{~Hz}$ \\
\hline bit & 256 \\
\hline effective aperture & $16 \times 12 \mathrm{~mm}$ \\
\hline fill factor & $95 \%$ \\
\hline
\end{tabular}

Table 4. The specification of the CMOS Camera.

\begin{tabular}{|l|l|}
\hline pixels & $1280 \times 1024$ \\
\hline pixel size & $5.2 \mathrm{um} \times 5.2 \mathrm{um}$ \\
\hline bit & 256 \\
\hline $\mathrm{S} / \mathrm{N}$ & $>54 \mathrm{~dB}$ \\
\hline Response & $1.8 \mathrm{~V} /($ Lux-sec) at $550 \mathrm{~nm}$ \\
\hline
\end{tabular}

In this experiment, defocus and tilt angle was varied simultaneously in $1.0 \mathrm{~mm}$ and $1.0 \mathrm{deg}$, respectively. The wavefront aberration introduced by lens 3 is expressed using Zernike polynomials. The amplitude of the aberration is represented using aberration vector. The wavefront pattern of defocus and tilt are shown in Fig. 7(a), (b), respectively. The wavefront pattern for compensation of the wavefront aberration is generated by LCOS-SLM as shown in Fig. 7(d). That pattern is generated as a superposition of that of defocus, tilt, and the inherent deformation of the LCOS-SLM that is shown in Fig. 7(c).



(a)



(c) (b)

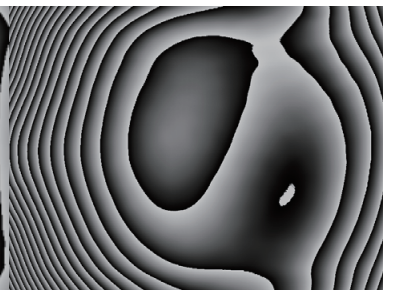

(d)
Fig. 7. The wavefront patterns: (a) Defocus, (b) tilt, (c) inherent deformation of LCOS-SLM, (d) total wavefront for compensating aberration as a superposition of $a, b$, and $c$.

The point spread function (PSF) with no defocus and no tilt angle is obtained as shown in Fig. 8(a). That is considered as the ideal PSF of no wavefront aberration. The PSF introduced by defocus and tilt is shown in Fig. 8(b), in which the PSF is extended 
asymmetry. Fig. 8(c) shows the compensated PSF using LCOS-SLM with wavefront shown in Fig. 7(d). The FWHM is defined in both $x-z$ plane and $y-z$ plane which include the pixel of maximum value as shown in Fig. 8(a-c); the $\mathrm{z}$ axis is defined as a pixel value direction. In this case, FWHM in y-direction is remarkably improved from $44.3 \mu \mathrm{m}$ to $33.4 \mu \mathrm{m}$ by wavefront compensation using LCOS-SLM, and the compensated PSF almost corresponds to that of no wavefront aberration shown in Fig. 8(a).
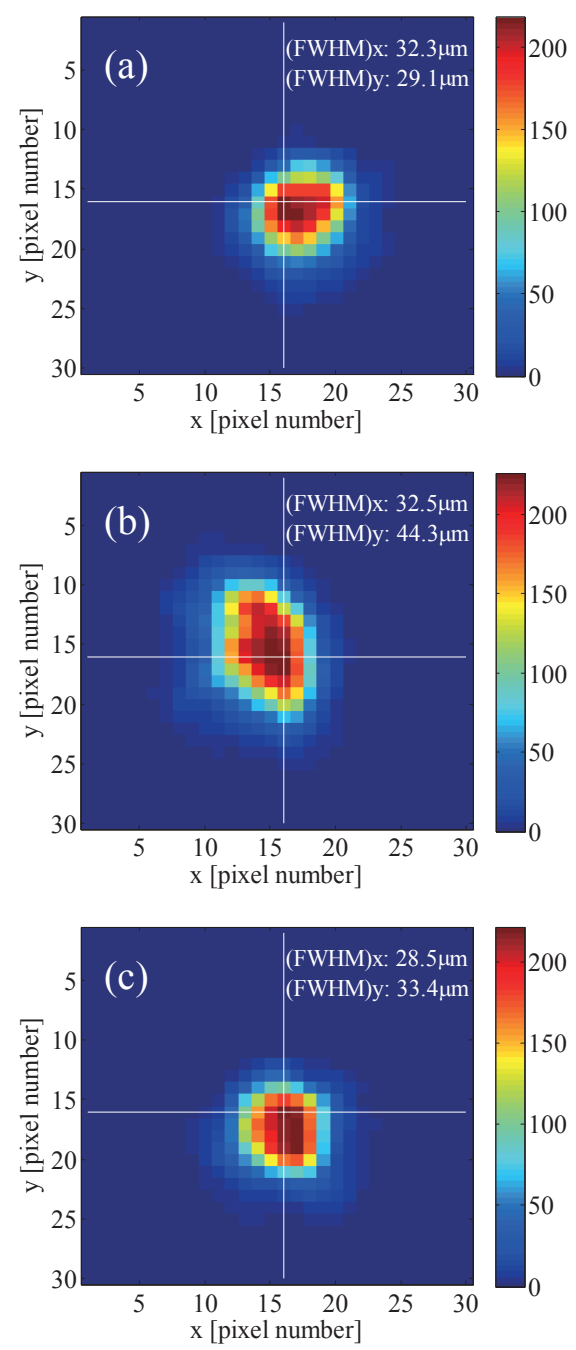

Fig. 8. Observed PSF: (a) Ideal PSF which is generated with no defocus and tilt, (b) PSF which is generated with defocus and tilt of converging lens, (c) compensated PSF using LCOS-SLM.

\section{Conclusion}

In this work, we proposed the wavefront aberration estimation method. The wavefront estimation is formulated as well-posed inverse problem by applying a priori information, which is defined as the phase diversity, to the optics system using the spatial light modulator. In this case, feature vectors are extracted from observed images then the neural network is used to solve the inverse problem for reduction of the computation cost. Since the response of the feature vector against the wavefront aberration depends on the applied phase diversities, we described the phase diversity selection strategy to determine the shape and the number of phase diversities. The numerical simulation is conducted to confirm the proposed wavefront estimation method. We also described the result of the wavefront compensation experiment. In the experiment, the wavefront aberration, which is introduced by defocus and tilt of the converging lens, is compensated using the LCOS-SLM and the quality of the observed image is improved. The results show that the LCOS-SLM has the ability of wavefront control for imaging system, and it can be used to compensate the wavefront aberration and to generate phase diversities.

\section{Acknowledgments}

This work was supported by JSPS KAKENHI 21360414.

\section{References}

1) Gonsalves, R.: Phase retrieval and diversity in adaptive optics, Opt. Eng., 21 (1982), pp. 829-832.

2) Blanc, A., Mugnier, L. and Idier, J.: Marginal estimation of aberrations and image restoration by use of phase diversity, $J$. Opt. Soc. Am. A, 20 (2003), pp.1035-1045.

3) Inoue, T., Tanaka, H., Fukuchi, N., Takumi, M., Matsumoto, N., Hara, T., Yoshida, N., Igasaki, Y. and Kobayashi, Y.: LCOS spatial light modulator controlled by 12-bit signals for optical phase-only modulation, Proc. SPIE, 6487 (2007), pp.64870Y_1-64870Y 11 .

4) Gruneisen, M., Dymale, R., Rotge, J., DeSandre, L. and Lubin, D.: Compensated telescope system with programmable diffractive optic, Opt. Eng., 44 (2005), pp.23201_1-23201_9.

5) Love, G.: Wave-front correction and production of Zernike modes with a liquid-crystal spatial light modulator, Appl. Opt, 36 (1997), pp. 1517-1524.

6) Andrews, J., Teare, S., Restaino, S., Wilcox, C., Wick, D., Xiao, H. and Schwiegerling, J.: Optical testbed for comparative analysis of wavefront sensors, Proc. SPIE, 5892 (2005), pp.589221_1-589221 7 .

7) Goodman, J.: Introduction to Fourier Optics, Roberts \& Company Publishers, 2004, pp.127-172.

8) Noll, R.: Zernike polynomials and atmospheric turbulence, $J$. Opt. Soc. Am. A, 66 (1976), pp. 207-211.

9) Born, M. and Wolf, E.: Principles of Optics, Cambridge University Press, 1999, pp.517-553.

10) Optical Research Associates: CODE V ver.10.0 Reference Manual, 2009, pp.10_21-10_24.

11) Paxman, R., Schulz, T. and Fienup, J.: Joint estimation of object and aberrations by using phase diversity, J. Opt. Soc. Am. A, 9 (1992), pp.1072-1085.

12) Kendrick, R., Acton, D. and Duncan, A.: Phase-diversity wave-front sensor for imaging systems, App.l Opt., 33 (1994), pp.6533-6546.

13) Miller, N. and Ling, A.: Imaging with phase diversity: simulations with a neural network, Proc. SPIE, 1982 (1993), pp.410-417. 